\title{
ON SEMIGROUPS OF OPERATORS IN LOCALLY CONVEX SPACES
}

\author{
FRODE TERKELSEN
}

1. The following problem is investigated in this note: Let $\{T(\xi)\}_{\xi>0}$ be a semigroup of continuous linear operators in a locally convex space $E$. Under which conditions (e.g. measurability conditions) is it true that the functions $\xi \rightarrow T(\xi) x$ are continuous for every $x \in E$ ? The point of departure is the paper [6] by Singbal-Vedak.

In [6], the following generalization of earlier results for Banach spaces by Dunford [1] and Hille [2] was proved: If $\{T(\xi)\}_{\xi>0}$ is a measurable semigroup such that $\{T(\xi)\}_{\alpha \leqq \xi \leqslant \beta}$ is equicontinuous for any $[\alpha, \beta] \subset(0, \infty)$, then the functions $\xi \rightarrow T(\xi) x$ are continuous. It is shown in Proposition 1 that a weaker equicontinuity condition, which is only required to hold locally at the origin 0 , implies the conclusion. Proposition 2 treats the special case of semigroups in LF spaces. Another theorem by Singbal-Vedak [6], generalizing a result of Phillips [4] for Banach spaces, says that if $E$ is a Fréchet space and the semigroup is measurable, then $\xi \rightarrow T(\xi) x$ is continuous for every $x \in E$. In Proposition 3 it is shown that the conclusion remains true when $E$ is merely a metrizable locally convex space.

2. The notation and definitions used in this note agree with [6]. We say that a semigroup of operators in a Hausdorff locally convex space $E$ is weakly measurable (or, almost separably valued) if, for each $x \in E$, the function $\xi \rightarrow T(\xi) x$ is weakly measurable (or, almost separably valued). Observe that a measurable function $f: R \rightarrow E$ is weakly measurable and almost separably valued, while the converse is known to hold if $E$ is metrizable (by a generalization of a result of Pettis [3]). It seems to be an open problem whether the converse holds for nonmetrizable $E$, or not.

In Proposition 1, $\tau\left(E, E^{\prime}\right)$ denotes the Mackey topology on $E$ (see e.g. [5]). Suppose $f: I \rightarrow E$ is a weakly measurable and almost separably valued function, where $I \subset R$ is a compact interval, and suppose $q$ is a $\tau\left(E, E^{\prime}\right)$-continuous seminorm on $E$. Then (i) $\xi \rightarrow q(f(\xi))$ is a measurable function, and (ii) for any $\epsilon>0$, there exists a continuous function $g: I \rightarrow E$ such that $\int_{I} q(f(\xi)-g(\xi)) d \xi<\epsilon$ (these facts are, with small modifications, contained in [6, Proposition 1 and the proof of Proposition 2]).

Received by the editors September 5, 1968. 
Proposition 1. Let $\{T(\xi)\}_{\xi>0}$ be a weakly measurable and almost separably valued semigroup of continuous linear operators in a locally convex space E, satisfying:

(I) for each neighborhood $U$ of 0 and each continuous seminorm $p$ on $E$, there exists a measurable set $A \subset U \cap(0, \infty)$ with $m(A)>0$ such that $\{p \circ T(\xi)\}_{\xi \in A}$ is an equicontinuous set of mappings of $\left(E, \tau\left(E, E^{\prime}\right)\right)$ into $R$.

Then $\xi \rightarrow T(\xi) x$ is continuous for every $x \in E$.

Proof. Let $x \in E$ and $\xi>0$. Given any continuous seminorm $p$ on $E$, we have to prove that $\lim _{\delta \rightarrow 0} p(T(\xi+\delta) x-T(\xi) x)=0$. Defining the point $\alpha=\xi / 3$, condition (I) implies the existence of a measurable set $A \subset(0, \alpha)$ with $m(A)>0$ and a $\tau\left(E, E^{\prime}\right)$-continuous seminorm $q$ on $E$ such that $p(T(\eta) y) \leqq q(y)$ for $\eta \in A$ and $y \in E$. Given $\epsilon>0$, choose a continuous function $g:[-\alpha, 2 \alpha] \rightarrow E$ such that

$$
\int_{-\alpha}^{2 \alpha} q(T(\xi-\eta) x-g(\eta)) d \eta<\epsilon
$$

(this is possible according to (ii) of the remark above). For any $\delta$ with $|\delta|<\alpha$ and any $\eta \in A$, we have

$$
\begin{aligned}
p(T(\xi+\delta) x-T(\xi) x) & =p(T(\eta)(T(\xi+\delta-\eta) x-T(\xi-\eta) x)) \\
& \leqq q(T(\xi+\delta-\eta) x-T(\xi-\eta) x) .
\end{aligned}
$$

Since the function $\eta \rightarrow q(T(\xi+\delta-\eta) x-T(\xi-\eta) x)$ is measurable according to (i) above, integration with respect to $\eta$ yields

$$
\begin{aligned}
m(A) p(T(\xi+\delta) x-T(\xi) x) \leqq & \int_{A} q(T(\xi+\delta-\eta) x-T(\xi-\eta) x) d \eta \\
\leqq & \int_{0}^{\alpha} q(T(\xi+\delta-\eta) x-g(\eta+\delta)) d \eta \\
& +\int_{0}^{\alpha} q(g(\eta+\delta)-g(\eta)) d \eta \\
& +\int_{0}^{\alpha} q(g(\eta)-T(\xi-\eta) x) d \eta<3 \epsilon,
\end{aligned}
$$

for sufficiently small values of $\delta$. Therefore the function $\eta \rightarrow T(\eta) x$ is continuous at the point $\xi$.

The following condition clearly implies that (I) of Proposition 1 holds: for each $[\alpha, \beta] \subset(0, \infty),\{T(\xi)\}_{\alpha \leq \xi \leq \beta}$ is equicontinuous (cf. $[6$, Proposition 2]). In (I), however, $A$ is allowed to depend on the 
seminorm $p$. The equicontinuity with respect to the $\tau\left(E, E^{\prime}\right)$-topology is in general a weaker condition than equicontinuity with respect to the given topology on $E$ (suppose, for example, that we consider $E$ with the weak topology $\left.\sigma\left(E, E^{\prime}\right)\right)$. Finally, (I) is a local condition at 0 .

In the next proposition, we consider an LF space, i.e. a strict inductive limit of an increasing sequence of Fréchet spaces (see [5]; the space $D$ of distribution theory is an LF space). It is known that an LF space has the $\tau\left(E, E^{\prime}\right)$-topology, and it is barrelled and nonmetrizable.

Proposition 2. Let $E$ be an $L F$ space with respect to the subspaces $\left(E_{n}\right)$, and let $\{T(\xi)\}_{\xi>0}$ be a weakly measurable and almost separably valued semigroup of continuous linear operators in $E$. Then $\xi \rightarrow T(\xi) x$ is continuous for every $x \in E$ if, and only if, for every $x \in E$ and every $[\alpha, \beta] \subset(0, \infty)$, there exists an $n \in N$ such that $\{T(\xi) x\}_{\alpha \leqq \xi \leqq \beta} \subset E_{n}$.

Proof. Assume $\xi \rightarrow T(\xi) x$ to be continuous. Then $\{T(\xi) x\}_{\alpha \leqq \xi \leqq \beta}$ is compact, and hence bounded in $E$, so it is contained in some $E_{n}$ $[5$, p. 59]. For the converse, suppose $p$ is a continuous seminorm on $E$ and $U$ is a neighborhood of 0 . Choose an interval $[\alpha, \beta] \subset U \cap(0, \infty)$. In order to verify that (I) of Proposition 1 holds, it is sufficient to show that $\{p \circ T(\xi)\}_{\alpha \leqq \xi \leq \beta}$ is pointwise bounded. For then the uniform boundedness principle for continuous seminorms, in the barrelled space $E$, implies that $\{p \circ T(\xi)\}_{\alpha \leqq \xi \leq \beta}$ is equicontinuous.

Suppose there exists an $x \in E$ such that $\{p(T(\xi) x)\}_{\alpha \leq \xi \leq \beta}$ is unbounded. Then we find a sequence $\left(\xi_{j}\right) \subset[\alpha, \beta]$ and a point $\gamma \in[\alpha, \beta]$ with $\lim _{j \rightarrow \infty} \xi_{j}=\gamma$ and $p\left(T\left(\xi_{j}\right) x\right) \geqq j$ for all $j \in N$. By assumption, there is an $n$ such that $\{T(\xi) x\}_{\alpha / 2 \leqq \xi \leqslant \beta} \subset E_{n}$. Let $\left(p_{k}\right)$ be a sequence of continuous seminorms on $E_{n}$ which defines the topology of $E_{n}$. For each $k$, there exists a continuous seminorm $p_{k}^{\prime}$ on $E$ such that $\left.p_{k}^{\prime}\right|_{E_{n}}=p_{k}$. Since $\xi \rightarrow p_{k}^{\prime}(T(\xi) x)$ of $(0, \infty) \rightarrow R$ is measurable, the restriction of the function $\xi \rightarrow p_{k}(T(\xi) x)$ to the interval $[\alpha / 2, \gamma]$ is measurable. From here on, proceed as in the proof of Proposition 3 of [6]. We find a set $F \subset[\alpha / 2, \gamma]$ such that $\{T(\xi) x\}_{\xi \in F}$ is a bounded subset of $E_{n}$, and a point $\sigma_{0} \in(0, \alpha / 2)$ satisfying the property: for each $j \in N$, there exists $\eta_{j} \in F$ with $\sigma_{0}=\xi_{j}-\eta_{j}$. Then $p\left(T\left(\sigma_{0}\right) T\left(\eta_{j}\right) x\right)$ $=p\left(T\left(\sigma_{0}+\eta_{j}\right) x\right)=p\left(T\left(\xi_{j}\right) x\right) \geqq j$ for each $j$, contradicting the fact that $T\left(\sigma_{0}\right)\left[\{T(\xi) x\}_{\xi \in F}\right]$ is bounded. We conclude that $\{p \circ T(\xi)\}_{\alpha \leqq \xi \leqq \beta}$ is pointwise bounded. By Proposition $1, \xi \rightarrow T(\xi) x$ is continuous for every $x \in E$.

Proposition 3. Let $\{T(\xi)\}_{\xi>0}$ be a measurable semigroup of continuous linear operators in a metrizable locally convex space $E$. Then 
$\{T(\xi)\}_{\alpha \leqq \xi \leqslant \beta}$ is equicontinuous for any $[\alpha, \beta] \subset(0, \infty)$, and $\xi \rightarrow T(\xi) x$ is continuous for every $x \in E$.

Proof. Let $\hat{E}$ denote the completion of $E$ and, for each $\xi>0$, let $\hat{T}(\xi): \hat{E} \rightarrow \hat{E}$ be the continuous extension of $T(\xi)$. Then $\{\hat{T}(\xi)\}_{\xi>0}$ is a semigroup of continuous linear operators in the Fréchet space $\hat{E}$. Given any $\hat{x} \in \hat{E}$, choose a sequence $\left(x_{n}\right)$ in $E$ such that $\lim _{n \rightarrow \infty} x_{n}=\hat{x}$. The functions $\xi \rightarrow \hat{T}(\xi) x_{n}=T(\xi) x_{n}$ are measurable by assumption, and for each $\xi>0$ we have $\lim _{n \rightarrow \infty} \hat{T}(\xi) x_{n}=\hat{T}(\xi) \hat{x}$. Therefore $\xi \rightarrow \hat{T}(\xi) \hat{x}$ is measurable, i.e. $\{\hat{T}(\xi)\}_{\xi>0}$ is a measurable semigroup. Now Proposition 3 of [6] implies that, for any $[\alpha, \beta] \subset(0, \infty),\{\hat{T}(\xi)\}_{\alpha \leq \xi \leq \beta}$ is equicontinuous, so $\{T(\xi)\}_{\alpha \leq \xi \leq \beta}$ is an equicontinuous set of mappings from $E$ into $E$. Hence, (I) of Proposition 1 is satisfied, so $\xi \rightarrow T(\xi) x$ is continuous for every $x \in E$.

\section{REFERENCES}

1. N. Dunford, On one parameter groups of linear transformations, Ann. of Math. (2) 39 (1938), 567-573.

2. E. Hille, Functional analysis and semi-groups, Amer. Math. Soc. Colloq. Publ., Vol. 31, Amer. Math. Soc., Providence, R. I., 1948.

3. B. J. Pettis, On integration in vector spaces, Trans. Amer. Math. Soc. 44 (1938), 277-304.

4. R. S. Phillips, On one-parameter semi-groups of linear transformations, Proc. Amer. Math. Soc. 2 (1951), 234-237.

5. H. H. Schaefer, Topological vector spaces, Macmillan, New York, 1966.

6. K. Singbal-Vedak, $A$ note on semigroups of operators on a locally convex space, Proc. Amer. Math. Soc. 16 (1965), 696-702.

Rutgers, The State University and University of Copenhagen 